\title{
The introduction of a homeless healthcare team in hospital improves staff knowledge and attitudes towards homeless patients
}

\author{
Authors: Vivienne Barrow ${ }^{\mathrm{A}}$ and Pippa Medcalf ${ }^{\mathrm{B}}$
}

It is estimated that 12,300 people slept rough in 2018 , a $98 \%$ increase since 2010. Similar trends can be seen in the number of people living in tents or sleeping on overnight public transport. Such individuals are five times more likely than age-matched, housed people, to attend hospital. This is due to the severe ill-health and poor engagement with primary care, prevalent among this population. Following the introduction of the Homeless Reduction Act 2017, hospitals must now take a more personalised approach to their homeless patients, ensuring that ongoing care is accessible after discharge. Here, we demonstrate that employment of a dedicated homeless housing officer within a district general hospital can radically improve both staff attitudes towards this patient group as well as individual patient outcomes.

KEYWORDS: Homeless, hospital, housing officer, drug, alcohol

\section{Introduction}

It is estimated that 12,300 people slept rough in 2018 , a $98 \%$ increase since 2010. Similar trends can be seen in the number of people living in tents or sleeping on overnight public transport. ${ }^{1}$ Eighty per cent of homeless individuals have at least one physical problem, $70 \%$ have mental ill health, ${ }^{2}$ and co-existing drug and alcohol dependence is common. ${ }^{2,3}$ However, this population is 40 times less likely to be registered with a general practitioner (GP). ${ }^{3}$ Consequently, the NHS spends an estimated $£ 85$ million each year on the care of the homeless; on average, a homeless patient costs eight times more than a housed patient. ${ }^{5}$ Despite this, the average age of death among this patient group is between 40 and 47 years old.

In a hospital setting, this hard to reach population ${ }^{6}$ presents with an additional acute problem, which often relates to their social circumstances. Physical injury, complications of drug use, alcohol related disease and infection are common presenting complaints but, owing to a frequently chaotic lifestyle, attendance is

Authors: ${ }^{A}$ general practitioner trainee, Newcastle upon Tyne Hospitals NHS Foundation Trust, Newcastle upon Tyne, UK; ${ }^{B}$ consultant, Gloucestershire Royal Hospital, Gloucester, UK commonly delayed until the problem has escalated. ${ }^{4,7}$ As a result, statistics from 2010 highlighted that, in comparison to the general population, emergency department (ED) attendance by the homeless was five times higher, inpatient admission was four times more common and the average stay was three times longer. ${ }^{3,5}$ In the subsequent 7 years this disparity has grown significantly; both ED attendance and admission rate have almost trebled, with the homeless population accounting for 31,274 hospital visits in England during the $2017 / 18$ financial year. ${ }^{9}$

There is evidence that the best indicator of patient health and successful resolution of homelessness is housing and good contact with specialist support agencies such as day centres, drugs and alcohol services and mental health support, who coordinate further referrals and help the patient to help themselves. ${ }^{3}$ Initial signposting to these services is important and creates the potential for community-based health management and reduced future reliance on the acute hospital. However, despite the frequent hospital contact, $70 \%$ of homeless patients are discharged without being offered any advice relevant to their homelessness. ${ }^{5}$ All too frequently, hospitals fail to recognise the link between unsafe discharge destination and a cycle of repeated readmissions with largely preventable illness, and discharge policies do not highlight homelessness as a 'red flag'. The Homelessness Reduction Act 2017 now mandates that all NHS trusts refer homeless patients to their local housing authorities prior to discharge, something which is still far from being achieved, at present. $^{8}$

It has been recommended that:

All acute hospitals are expected to implement formal policies which provide a framework to ensure the identification of homeless individuals as soon as they are admitted to hospital, so that community health care and tertiary sector homelessness services are notified prior to the patient's discharge. ${ }^{10}$

The University College Hospital NHS Trust has successfully implemented a pioneering strategy to address the issue surrounding the admission and discharge of homeless patients. 'Pathway' has achieved a remarkable $30 \%$ reduction in admissions, while saving over $£ 100,000$. $^{11}$ This highlights that the implementation of a local homeless policy is beneficial to the individual at hand, the staff looking after them and the NHS as a whole. 


\section{Materials and methods}

In 2013, we audited the management of homeless patients at the Gloucestershire Royal Hospital.

We studied the medical documentation for homeless individuals treated and discharged from ED $(n=25)$ and those discharged following admission to a ward $(n=25)$, in order to examine the discharge planning and outcome.

We also explored staff knowledge and attitudes towards homeless patient discharge by means of a 10 question multiple choice and blank space paper questionnaire. This was completed by members of the multidisciplinary team including doctors (representing a cross section of grades), nurses and other allied health professionals.

In 2016, the same questionnaire was redistributed to reassess staff attitudes in ED and medical assessment unit (MAU) following the introduction of a new policy for homeless patient discharge. Again, it was completed by doctors, nurses and other allied health professionals.

\section{Results - 2013}

In 2013 we identified that the hospital policy did not require homeless patients to be discharged to a place of safety and $78 \%$ of staff did not know what the hospital's policy was (Fig 1). Analysis of case notes highlighted that although all patients were identifiably homeless from the outset, discharge planning was not considered until the patient was medically fit. While $87 \%$ of staff believed referral to social work to be the correct course of action, referrals were always rejected as homelessness was not in their remit. Only one out of 50 patients was referred for housing advice and a there was a lack of sign-posting to tertiary services.

Sixty-eight per cent of patients were discharged to an unknown location and $78 \%$ of staff from ED and the acute medical unit (AMU) stated that patients were routinely discharged with nowhere to go (Fig 2).

Some staff were frustrated that more could not be done but time and bed pressures were the key factors seen to limit the possibility for sign-posting or planned discharge into accommodation. Many staff did not consider it to be the hospital's responsibility to sort out what was considered to be a social issue.

\section{Intervention}

As current practice was not in line with the Department of Health's guidelines, we consulted with the local community homeless healthcare team, who provide primary care and drop-in services to homeless individuals in Gloucester. Agreeing that a hospital inreach service at the point of admission would create an invaluable link between primary and secondary care and ensure appropriate sign-posting and coordinated discharge planning, we applied for and successfully obtained a start-up grant of $£ 34,000$ from the 2012 Department of Health initiative to support hospitalhomeless healthcare projects. This provided funding to employ a full-time housing officer and a part-time nurse for 1 year. An acute consultant physician volunteered her time to coordinate and promote the service.

The housing officer was employed to work weekdays from $9 \mathrm{am}-5 \mathrm{pm}$. He had a dedicated mobile phone and the telephone number was widely publicised with posters in every hospital department. Educational updates were also held to maximise awareness among hospital staff.

In response to receipt of a referral, the housing officer would conduct a same day patient review and assessment, arrange temporary accommodation and, perhaps most importantly, personally escort the patient to their accommodation. Input by the housing officer would continue in order to achieve more permanent accommodation through the council. He also arranged appropriate referrals with agencies such as drug and alcohol services, the Department for Work and Pensions, the dentist, chiropodist etc. The specialist nurse identified ongoing medical issues, arranged registration with a GP or the local homeless healthcare clinic, arranged follow-up and oversaw the supply and taking of medications.
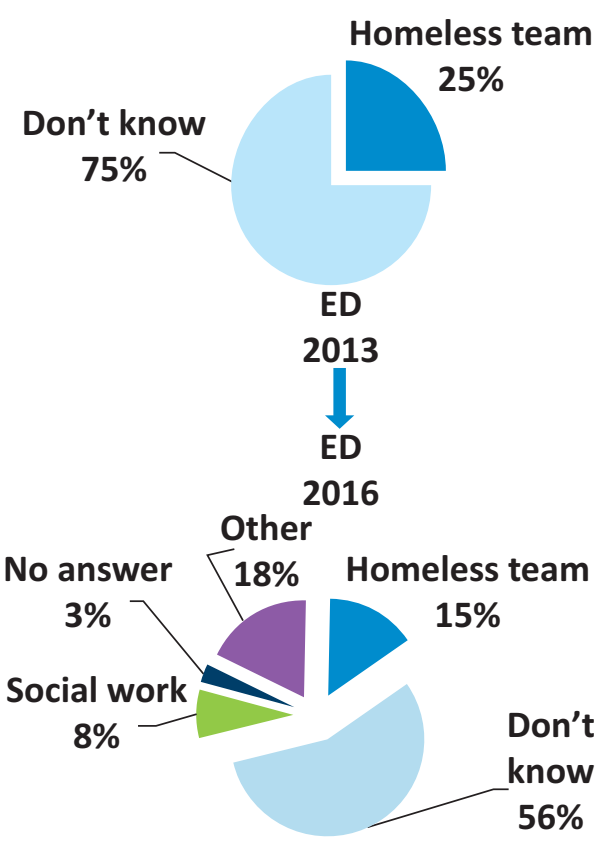

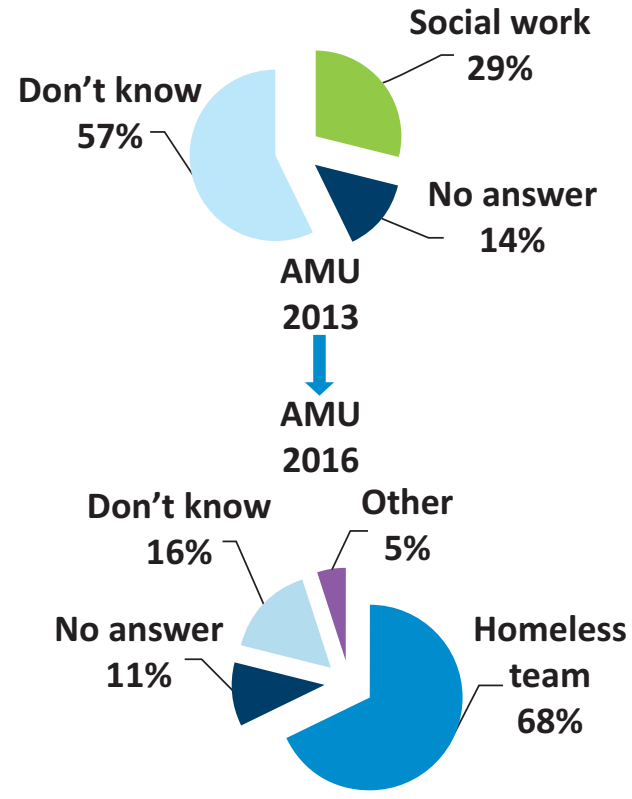

Fig 1. What is the hospital's homelessness policy? AMU = acute medical unit; $E D=$ emergency department. 
Fig 2. Is a discharge solution usually found? $\mathrm{AMU}=$ acute medical unit; $\mathrm{ED}=$ emergency department.

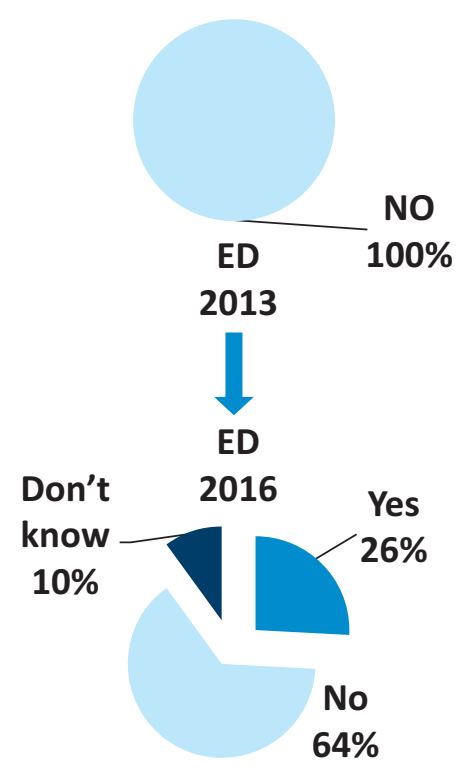

Initial outcomes following intervention - 2014

Our initial re-audit highlighted that within the first 6 months of implementation, 57 (54 first-time) referrals were made to the new hospital homeless team (HHT; $56 \%$ men, mean age 43 years) and $98 \%$ of patients were seen within 24 hours. Eighty-two per cent were discharged into housing (most commonly bed-and-breakfast places and hostels) and $80 \%$ patients were referred to drug and alcohol services compared to $4 \%$ previously. The mean length of stay reduced from 2.2 to 2.1 days. Discussion between staff and patients regarding accommodation increased from $10 \%$ to $79 \%$. In the first 12 months, the hospital observed an approximate saving of $£ 50,000$ from the care of this patient group.

Based on compelling evidence of the success of the first year's pilot, combined with local and national publicity which boosted support for the initiative, a business case was compiled and presented to the local clinical commissioning group (CCG). After several meetings, it was agreed that $£ 28,000$ funding would be allocated per annum towards the scheme. This was sufficient to fund the housing officer but not the nurse.

\section{Re-audit - 2016}

Three years after implementation, we re-audited staff knowledge and attitudes, using the same questionnaire. This highlighted a trend towards active engagement with the $\mathrm{HHT}$, with $68 \%$ of AMU staff now recalling this as the new hospital policy (Fig 1). When asked whether homelessness was hospital's responsibility, the percentage of staff responding 'yes' rose from $0 \%$ to $56 \%$ in ED and from $29 \%$ to $74 \%$ in AMU (Fig 3). The proportion of staff
Fig 3. Is homelessness a hospital's responsibility? $\mathrm{AMU}=$ acute medical unit; $\mathrm{ED}=$ emergency department.

Blank

\section{$25 \%$}

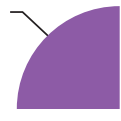

2013

1

Not sure 2016

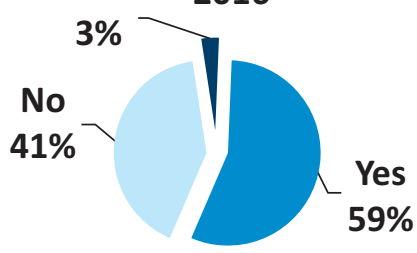

Blank
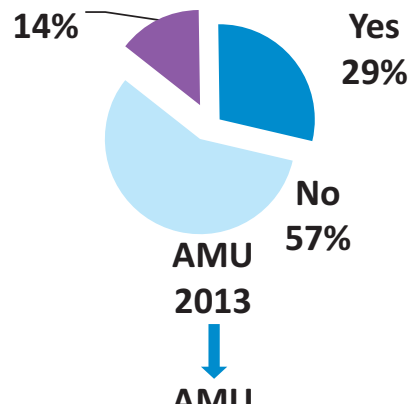

$29 \%$

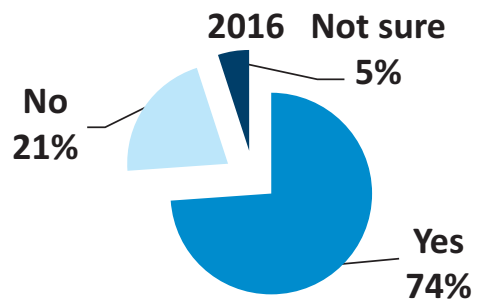




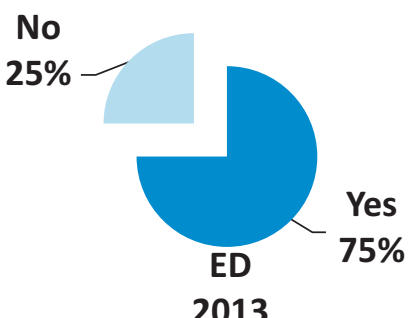

2013

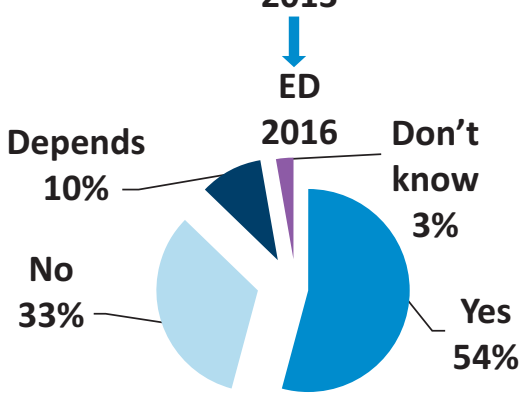

Don't know

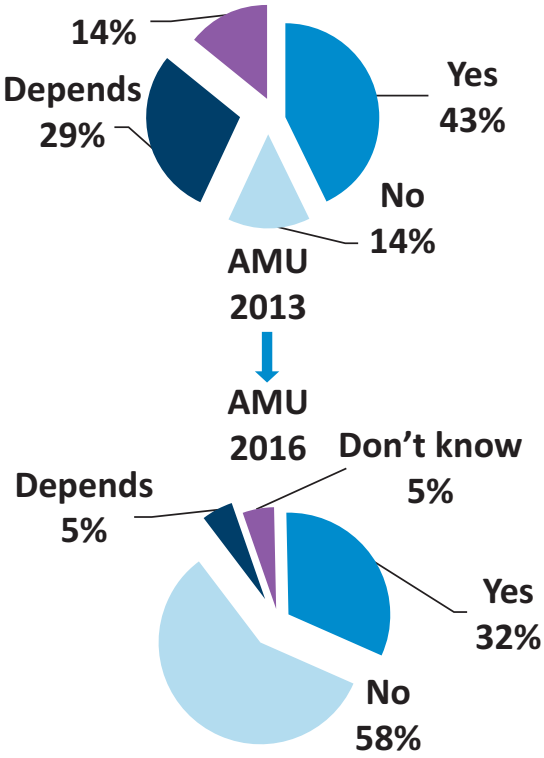

Fig 4. Should homeless patients be discharged with nowhere to go? AMU = acute medical unit; $E D=$ emergency department. unwilling to discharge a homeless patient without a discharge destination, increased from $25 \%$ to $33 \%$ in ED and from $14 \%$ to $58 \%$ in $\mathrm{AMU}$ (Fig 4). Following introduction of the HHT, 26\% ED staff believed that a housing solution was found on discharge, compared to $0 \%$ previously. In AMU, this increased from $29 \%$ to $53 \%$ (Fig 2).

\section{Discussion}

Homelessness is a frequently overlooked cause of preventable ill health. The homeless population is significantly over-represented in the hospital setting. Considering that these individuals are usually hard to reach, the hospital is a valuable point of contact and its role in initiating referral cannot be over-emphasised. Evidence points towards effective collaboration between hospitals and homelessness service providers as a solution to revolving door admissions, realising the potential not only to combat the significant burden of health inequality within this population but also to reduce unnecessary use of NHS services. It is estimated that each homeless patient costs the UK £20,000 per year. In contrast, successful intervention and rehabilitation has an estimated price tag of just £1,426 - an unarguably significant saving. $^{12}$

In light of the government's strategy to halve rough sleeping by 2022 and end it completely by 2027, together with the implementation of the Homelessness Reduction Act in 2018 which mandates public services, including hospitals, to initiate referrals to appropriate support agencies, our audit highlights that a simple strategy involving coordinated in-reach between primary and secondary care providers, which is initiated as soon as a patient is admitted, can effectively and efficiently achieve patientcentred and holistic care without prolonging hospital stay. The management of homeless patients may be perceived by staff as complex or time consuming especially in an acute hospital setting such as ED or AMU. Often the task of addressing a patient's homelessness is crowded out by the constant stream of other tasks requiring attention from healthcare staff. Our results show that implementation of a hospital-based homeless healthcare team, accessed by a single telephone referral, is well accepted by staff and improves homeless patient outcome, promoting a positive attitude towards the management of this patient group.

\section{Acknowledgements}

With many thanks to Dr Rose Glennerster, Dr Kitty Acheson, Dr Anduvan Andiran, Dr Felicity Cooksey and Dr Sarah Kyle who helped with data collection.

\section{References}

1 Butler P. 24,000 sleeping rough or on public transport in UK, charity says. Guardian, 2018. www.theguardian.com/society/2018/dec/13/ disgrace-rising-english-homelessness-includes-120000-children.

2 Homeless Link. The health and wellbeing of people who are homeless: Evidence from a national audit. London: Homeless Link, 2010

3 The Queen's Nursing Institute Homeless Health Initiative. Improving healthcare for homeless people - a learning resource. Section B, Module 1: Overview of health and homelessness 2010. QNI, 2010

4 Wright NM, Tompkins CN. How can health services effectively meet the health needs of homeless people? Br J Gen Pract 2006;56:286-93.

5 Department of Health. $£ 10$ million funding for hospital aftercare for the homeless. London: DH, 2013. www.gov.uk/government/ news/10million-funding-for-hospital-aftercare-for-the-homeless [Accessed 18 September 2018].

6 Lewis C. Equality Compliance Report. Gloucestershire Hospitals NHS Foundation Trust, 2011.

7 Communities and Local Government, Department of Health. Hospital Admission and Discharge: People who are homeless or living in temporary or insecure accommodation. Wetherby: Communities and Local Government Publications, 2006.

8 Kennedy I. The 'duty to refer': why the CQC should review their inspection regime. London: Royal College of Physicians, 2018. www.rcplondon.ac.uk/news/duty-refer-why-cqc-should-review-theirinspection-regime [Accessed 12 Mach 2019]. 
9 Blackburn P. Streets of shame: homelessness and the NHS - tomes of tragedies. Doctor 2019:7-13. www.bma.org.uk/features/ streetsofshame [Accessed 11 March 2019].

10 Homeless Link, St Mungo's. Improving hospital admission and discharge for people who are homeless: Analysis of the current picture and recommendations for change. Homeless Link, St Mungo's, 2012.

11 University College London Hospitals. Helping the homeless. UCLH, 2012. www.uclh.nhs.uk/News/Pages/28Helpingthehomeless5.aspx [Accessed 18 September 2018].
12 Pleace N. At what cost? An estimation of the financial costs of single homelessness in the UK. York: University of York, 2015. www.crisis.org.uk/media/237022/costsofhomelessness_finalweb.pdf [Accessed 12 March 2019].

Address for correspondence: Dr Pippa Medcalf, Gloucestershire Royal Hospital, Great Western Road, Gloucester, Gloucestershire GL1 3NN, UK.

Email: vivbarrow2112@gmail.com Treating tobacco dependency in the NHS

Published in 2018, this report by the Tobacco Advisory Group of the Royal College of Physicians addresses the harms and costs arising from smoking in the patients we see every day, and argues for a new approach to treating their addiction.

Download the report at: www.rcplondon.ac.uk/hidingplain-sight-treating-tobaccodependency-nhs

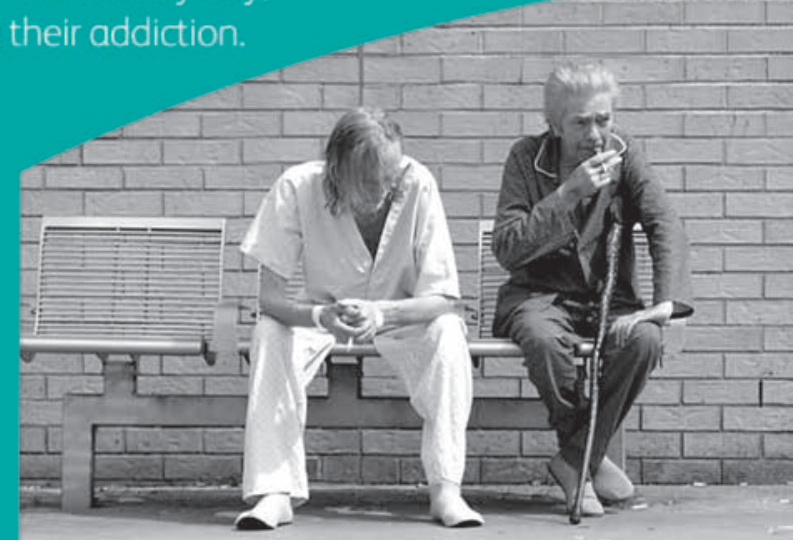

